\title{
El argumento del objeto del pensamiento en el tratado aristotélico Sobre las Ideas (Peri ideōn)
}

\author{
JosÉ EDGAR GONZÁLEZ VARELA \\ Department of Philosophy \\ University of Sheffield \\ pip05jeg@sheffield.ac.uk
}

\begin{abstract}
Resumen: En el tratado aristotélico Sobre las Ideas (Peri ideōn) hay cinco argumentos platónicos a favor de la existencia de Ideas. Según Metafísica A 9, dos de estos argumentos son "más rigurosos" que los otros. La interpretación estándar de esta distinción sostiene que los argumentos "más rigurosos" son válidos para postular Ideas, mientras que los "menos rigurosos" no lo son. En este trabajo cuestiono esta interpretación, pues ofrezco una nueva interpretación de uno de los argumentos "menos rigurosos", el argumento del objeto del pensamiento, según la cual éste es válido para postular Ideas.
\end{abstract}

Palabras clave: universales, separación, Platón

\begin{abstract}
There are five platonic arguments for the existence of Ideas in Aristotle's treatise On Ideas (Peri ideōn). According to Metaphysics A 9 two of these arguments are "more accurate" than the others. The standard interpretation of this distinction claims that the "more accurate" arguments are valid to conclude the existence of Ideas while the "less accurate" ones are not. In this paper I call into question this interpretation, for I offer a new interpretation of one of the "less accurate" arguments, the object of thought argument, according to which this is valid to conclude the existence of Ideas.
\end{abstract}

Key words: universals, separation, Plato

\section{Introducción}

El tratado Sobre las Ideas (Peri ideōn) es una de las obras de Aristóteles que, desafortunadamente, no ha llegado hasta nosotros de manera directa y completa. En ese tratado Aristóteles se ocupa de discutir algunas cuestiones relacionadas con la teoría platónica de las Ideas y con la teoría de las Ideas de Eudoxo, filósofo académico. Lo que sabemos del Peri ideōn proviene del comentario de Alejandro de Afrodisia (siglos IIIII d.C.) a la Metafísica de Aristóteles. En Metafísica A 9 (990b8-17), Aristóteles menciona escuetamente lo siguiente: (i) cinco argumentos platónicos a favor de las Ideas, y sus críticas a éstos, (ii) un grupo de argumentos que cuestionan la consistencia entre los argumentos platónicos para postular Ideas y la doctrina platónica de los principios, y 
(iii) una crítica a la teoría de las Ideas de Eudoxo. Alejandro, intentando aclarar estas breves alusiones, presenta en su comentario los tres grupos de argumentos con más amplitud y detalle. Es incierto hasta qué punto el material del Peri ideōn contenido en el comentario de Alejandro constituye una transcripción más o menos exacta del tratado aristotélico y en qué medida se trata de una paráfrasis suya. También es incierto determinar el contenido preciso del tratado, si sólo contenía estos argumentos, o si su extensión era mayor. Sin embargo, hay consenso en que existen buenas razones para afirmar que Alejandro presenta, al menos, una relación bastante fiel de una parte del tratado aristotélico. $^{1}$

El Peri ideōn constituye la primera disputa registrada en la historia de la filosofía occidental sobre el problema de los universales. ${ }^{2} \mathrm{El}$ problema de los universales es el de explicar la identidad de naturaleza que cosas numéricamente distintas parecen compartir. Por ejemplo, esta lámpara blanca y esta flor blanca parecen tener algo en común, parecen ser idénticas en algún aspecto de su naturaleza: en su blancura. El problema consiste en averiguar si esta supuesta identidad de naturaleza es sólo aparente o si es real; esto es, si existen o no propiedades y/o relaciones que sean objetivas en el sentido de ser independientes de la mente que las piensa y del lenguaje en que son expresadas.

Aristóteles y Platón son realistas con respecto al problema de los universales, pues concuerdan en que hay universales objetivos; no obstante, difieren en algunos de los rasgos que les atribuyen a éstos. Su principal punto de desacuerdo es la separación, la cual es el rasgo distintivo de las Ideas o universales platónicos, y consiste en la capacidad que, según Platón, los universales tienen de poder existir sin ser instanciados. En este sentido, se puede decir que los universales platónicos son independientes ontológicamente de los particulares que los instancian. $^{3}$ Aristóteles, por su parte, rechaza este rasgo de los universales platónicos, pues considera que para que un universal exista es necesario que sea instanciado; esto es, los universales aristotélicos son ontológicamente dependientes de las cosas que los instancian. Según Aristóteles,

${ }^{1}$ Una razón es que Alejandro es un comentarista cuidadoso en su uso de fuentes aristotélicas. Fine (1993, pp. 29-34) ha dado otras razones que apoyan la confiabilidad del texto.

${ }^{2} \mathrm{Al}$ parecer, el Peri ideōn es una de las primeras obras de Aristóteles, escrita probablemente siendo aún miembro de la Academia. Fine (1993, pp. 41-43) defiende convincentemente esta tesis.

${ }^{3}$ Sobre esta interpretación de la separación, véase Fine 1984, pp. 33-45, y Vlastos 1987. Devereux 1994 cuestiona esta lectura. 
un universal no puede ser ontológicamente independiente porque sólo las sustancias pueden tener tal independencia, y todas las cosas que son sustancias son particulares. Entonces, si un universal tuviera independencia ontológica, sería universal y particular al mismo tiempo; pero, según Aristóteles, nada puede ser universal y particular a la vez. ${ }^{4}$

Los argumentos del Peri ideōn más importantes, histórica y filosóficamente, son los cinco argumentos del primer grupo. Éstos son: los argumentos de las ciencias, el argumento de lo uno sobre muchos, el argumento del objeto del pensamiento, el argumento de los relativos y el argumento del tercer hombre. Éstos son argumentos platónicos a favor de la existencia de Ideas, los cuales son acompañados por críticas de Aristóteles a cada uno de ellos. ${ }^{5}$ Tanto en el Peri ideōn como en Metafísica A 9, Aristóteles sostiene que ninguno de estos argumentos es exitoso. No obstante, en Metafísica A 9 Aristóteles distingue entre ellos, pues afirma que dos, el argumento de los relativos y el del tercer hombre, son "más rigurosos" ( $\alpha x p ı \beta \varepsilon ́ \sigma \tau \varepsilon \rho ı$ ) que los tres restantes: los argumentos de las ciencias, el de lo uno sobre muchos y el del objeto del pensamiento. La interpretación estándar de esta distinción ${ }^{6}$ sostiene que ésta consiste en que los argumentos "menos rigurosos" no son válidos para postular Ideas, sino que sólo son válidos para postular universales aristotélicos, mientras que los "más rigurosos" son válidos para postular Ideas, pero no son sólidos. ${ }^{7}$

${ }^{4}$ Cfr., Metafísica Z 1. 1028a33-4, $\Delta$ 11. 1019a1-4, y M 9. 1086a31-b11. Véase, además, Fine 1984, pp. 38-39, 45. El argumento de Aristóteles me parece discutible. Una teoría de universales que aceptara universales de orden superior podría explicar cómo un universal puede ser universal y particular a la vez, siempre que no lo fuera con respecto al mismo orden (de manera semejante a la teoría de los tipos de Russell).

${ }^{5}$ Se ha debatido sobre si estos argumentos son genuinamente platónicos o si reflejan el punto de vista de algún otro filósofo académico, pues el autor no es explícitamente identificado por Alejandro. Sin embargo, Fine (1993, pp. 34-36) ha dado buenas razones para afirmar que éstos están inspirados en los diálogos platónicos del período medio y que, por tanto, el blanco de las críticas aristotélicas es la teoría platónica de las Ideas de ese período. Otros autores están en desacuerdo; por ejemplo, Isnardi Parente (1981) sostiene que el blanco de las críticas de Aristóteles no es Platón, sino Jenócrates.

${ }^{6}$ Así, Alejandro 78. 1-25, en Dooley 1989; Fine 1993, pp. 25-27; y Santa Cruz, Crespo y Di Camillo 2000, pp. 24-26 y 32-35.

${ }^{7}$ Un argumento es válido cuando su conclusión se sigue de sus premisas, esto es, cuando la verdad de las premisas garantiza la verdad de la conclusión. Una manera usual de expresar esta idea es decir que un argumento es válido si es imposible que sus premisas sean verdaderas y su conclusión sea falsa. Por otra parte, un argumento sólido, además de ser válido, tiene sólo premisas verdaderas. 
El objetivo principal de este trabajo es cuestionar esta interpretación y, en última instancia, la relevancia de la distinción misma entre argumentos "más y menos rigurosos" para el estudio del Peri ideōn. Intentaré mostrar que uno de los argumentos "menos rigurosos", el argumento del objeto del pensamiento, sí es válido para postular Ideas, y lo es porque de sus premisas se sigue la conclusión de que hay universales separados, $\mathrm{y}$, como he señalado, la separación es el rasgo distintivo de los universales platónicos. ${ }^{8}$ Sostengo que el argumento parte del supuesto de la existencia de universales objetivos y sólo intenta mostrar que éstos están separados. ${ }^{9}$

Pero si uno de los argumentos "menos rigurosos" del Peri ideōn es válido para postular Ideas, entonces la interpretación usual de la distinción aristotélica entre argumentos "más y menos rigurosos" es incorrecta, pues no es posible establecerla con base en la supuesta invalidez de los argumentos "menos rigurosos". No pretendo desarrollar en este trabajo una interpretación alternativa de dicha distinción, sino simplemente sugerir que ésta no debería ser tomada como el eje fundamental de interpretación de los cinco argumentos del Peri ideōn. La razón es que, a mi juicio, no se puede establecer una distinción sustantiva entre ambos grupos de argumentos, pues me parece que sólo podría haber una distinción importante en dos casos: en cuanto a validez o en cuanto a solidez. Pero espero mostrar en este trabajo que no es posible establecer una distinción en cuanto a la validez de los argumentos; y tampoco se puede establecer en cuanto a solidez, pues es evidente que para Aristóteles ninguno de ellos es sólido. Pienso que la distinción que Aristóteles establece de pasada en Metafísica A 9 consiste, muy probablemente, en una distinción secundaria que no es esencial para el

${ }^{8}$ Caston (1995, pp. 163-165) ha cuestionado también la interpretación tradicional del Peri ideōn. Caston sugiere que el argumento del objeto del pensamiento y los argumentos de las ciencias son válidos para postular Ideas, pero no extrae de esta tesis ninguna conclusión sobre el estatus de la distinción menos-más rigurosos. La interpretación de Caston (1998, pp. 264-265) del argumento del objeto del pensamiento es diferente de la que propongo en este trabajo (véase más adelante la n. 22).

${ }^{9}$ Así pues, según mi interpretación, el argumento no intenta mostrar ni la existencia ni la objetividad de los universales, sino que sostiene que si asumimos que hay universales y que éstos son objetivos, es decir, independientes de la mente y del lenguaje, entonces debemos concluir que éstos están separados. Esto es, el argumento presupone la verdad del realismo acerca de los universales, para ofrecer razones a favor de un tipo particular de realismo, el realismo de universales separados (realismo platónico).

Diánoia, vol. LIII, no. 60 (mayo 2008). 
análisis de los argumentos. ${ }^{10}$ Conjeturo que la distinción aristotélica obedece a que los argumentos "más rigurosos" lo son porque, quizá, a él le parece que tienen una mayor complejidad en sus premisas y en la articulación de las mismas. Y en efecto, tanto el argumento de los relativos como el del tercer hombre son argumentos más rebuscados y sutiles, pero esto no implica que sean "más rigurosos" que los otros en algún sentido importante.

En la siguiente sección presentaré el texto griego del argumento y una traducción mía. Después, en la tercera sección analizaré con detalle el argumento y mostraré por qué es válido para postular Ideas. En las secciones cuarta y quinta estudiaré las críticas aristotélicas al argumento y defenderé que éstas intentan cuestionar sólo su solidez, pero no su validez. ${ }^{11}$ Sostengo que Aristóteles está en lo correcto, que el argumento del objeto del pensamiento no es sólido; esto es, aunque la existencia de Ideas se sigue válidamente de sus premisas, al menos una de éstas es, a mi juicio, falsa. Por tanto, considero que este argumento debe ser abandonado por cualquier realista de universales que busca defender su separación ontológica.

\section{Texto y traducción ${ }^{12}$}

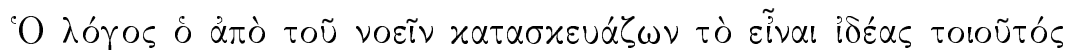

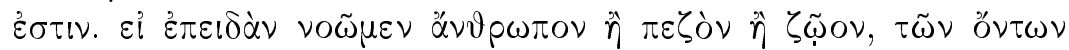

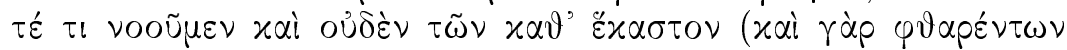

${ }^{10}$ Otra razón para dudar de la importancia de la distinción de Metafísica A 9 para interpretar los argumentos del Peri ideōn puede extraerse del hecho muy probable de que tal distinción no se haya encontrado en esta última obra, pues Alejandro, quien seguramente conocía este tratado de primera mano, tiene serias dificultades para explicar en qué consistía tal distinción. Véase Alejandro 78. 1-25, en Dooley 1989. Otros autores han notado este punto: Santa Cruz, Crespo y Di Camillo 2000, pp. 20-21, y Düring 1990, p. 390.

${ }^{11}$ Aristóteles introduce explícitamente dos críticas contra el argumento, las cuales, como veremos, cuestionan su solidez, pero no su validez. Ahora bien, se debe señalar que existen dos recensiones del texto del Peri ideōn, la recensio vulgata (OAC) y la recensio altera (LF). OAC es más confiable que LF y es el texto que los estudiosos de este tratado traducen y analizan. Es en OAC donde no aparece una objeción a la validez del argumento. No obstante, tal objeción sí aparece en LF. Sobre esta evidencia y sobre el uso de LF para interpretar el Peri ideōn hablaré al inicio de la sección 4. El texto que he empleado es el de OAC; sin embargo, incluyo la parte relevante de LF y una traducción de la misma.

${ }^{12}$ El texto griego empleado es el de Fine 1993, quien reproduce con pocas variaciones el de Harlfinger 1975. 


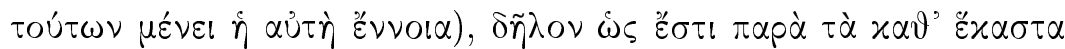

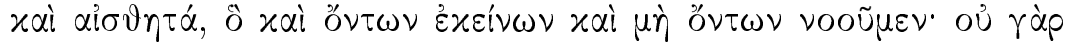

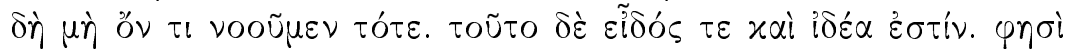

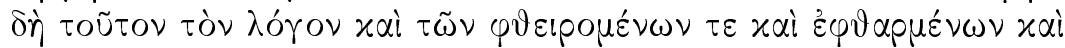

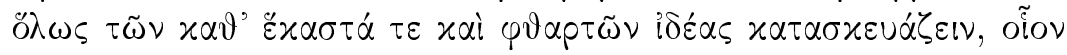

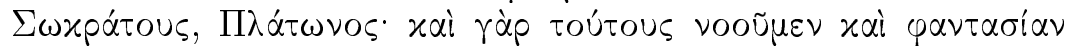

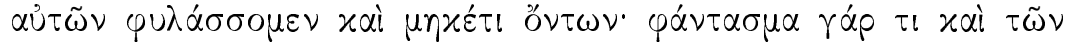

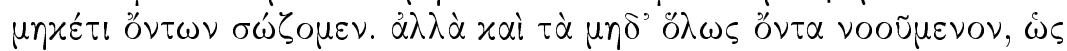

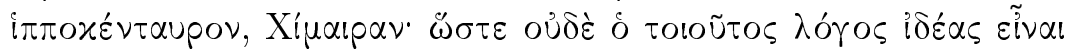

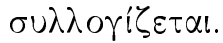

\section{[Adición de LF]}

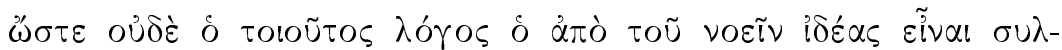

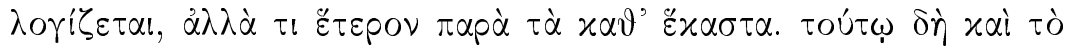

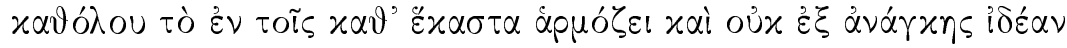

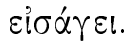

El argumento que establece que hay Ideas a partir del pensar es el siguiente:

Si cuando pensamos en hombre, pedestre o animal, pensamos en alguna de las cosas que son y en ninguna de las cosas particulares (pues el mismo pensamiento permanece incluso cuando éstas se han destruido), es evidente que existe [algo] aparte de las cosas particulares y sensibles, en lo cual pensamos tanto si aquellas son como si no son. En efecto, ciertamente no pensamos en este caso algo que no es. Pero esto es una Forma o Idea.

Dice Aristóteles que este argumento establece Ideas de las cosas que se destruyen y de las que están destruidas y, en general, de las cosas particulares y destructibles, como Sócrates o Platón. En efecto, también pensamos en estas cosas y retenemos una imagen de éstas, incluso cuando ya no son. En efecto, conservamos una imagen de las cosas que ya no son. Pero también pensamos en las cosas que no son en absoluto, como en hipocentauro o Quimera. De modo que tampoco este argumento concluye que hay Ideas.

\section{[Adición de LF]}

[De modo que tampoco este argumento a partir del pensar concluye que hay Ideas, sino que hay algo otro aparte de las cosas particulares. Ciertamente, el universal que está en los particulares también se ajusta a esto, y no necesariamente introduce una Idea. $]^{13}$

\footnotetext{
${ }^{13}$ Sobre esta adición de (LF), véase la n. 11 y el inicio de la sección 4.
} 


\section{El argumento del objeto del pensamiento}

En esta sección analizo el argumento del objeto del pensamiento. Sostengo que éste es válido para postular Ideas, pues de sus premisas se sigue válidamente la conclusión de que hay universales separados; y la separación es el rasgo distintivo de las Ideas.

A continuación presento una formulación preliminar del argumento del objeto del pensamiento, la cual será útil como punto de partida del análisis, pues se ciñe estrictamente al texto del Peri ideōn. Sin embargo, más adelante ofreceré una formulación más depurada en la que espero pueda apreciarse con mayor claridad la forma lógica del argumento y, por ende, su validez. Así pues, el argumento es el siguiente:

(1) Si pensamos en hombre, pedestre o animal, entonces pensamos en alguna de las cosas que son.

(2) El mismo pensamiento permanece incluso cuando las cosas particulares se han destruido.

(3) Si pensamos en hombre, pedestre o animal, entonces no pensamos en alguna de las cosas particulares.

(4) Existe algo aparte de las cosas particulares y sensibles, en lo cual pensamos tanto si éstas son como si no son.

(5) Esto es una Forma o Idea.

(6) Existen las Ideas.

El verbo principal del argumento, voعiv, lo he traducido como "pensar". Sin embargo, vогĩ puede significar también "conocer" o "entender". ${ }^{14}$ Creo que "pensar" es la traducción más adecuada, en primer lugar, porque es menos restrictiva, pues este término puede designar distintos tipos de actividades mentales como creer, considerar o concebir. "Conocer" o "entender" denotan un tipo de pensamiento, pero conllevan connotaciones adicionales como las condiciones de éxito, verdad y justificación. Además, en el Peri ideōn ya hay un argumento que intenta postular Ideas por consideraciones epistemológicas: los argumentos de las ciencias. Por tanto, parece plausible sostener que este argumento proporciona razones distintas para postular Ideas. No obstante, es preciso reconocer que hay importantes similitudes entre el argumento del

${ }^{14}$ Cfr. Liddell, Scott y Jones 1968, p. 1177. En Platón, el segundo sentido de este verbo predomina en República VI y VII. El primero se ejemplifica en Fedón 73c. 
objeto del pensamiento y los argumentos de las ciencias, pues el primero podría formularse como un argumento del objeto del conocimiento y el resultado sería un argumento diferente pero todavía platónico. Creo que esto indica que ambos argumentos comparten, con diferentes matizaciones, una tesis común: sólo es posible pensar/conocer aquello que es. ${ }^{15}$ Con todo, por las razones expuestas me parece más conveniente interpretar en este argumento el verbo voعĩ como "pensar", pero también puede ser útil mantener los otros significados en mente en este trabajo. ${ }^{16}$

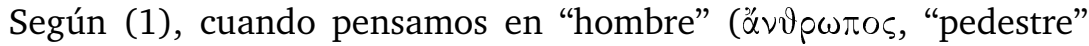

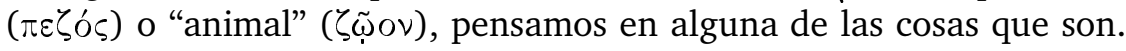
(1) puede tener sentidos distintos dependiendo de cómo se entienda

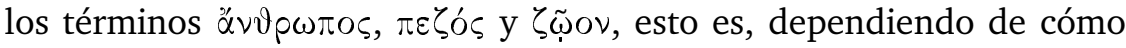
se interprete aquello que (1) identifica como objeto de pensamiento. (a) Una primera opción sería interpretar que estos términos son usados para referir indefinidamente a cosas particulares (un uso frecuente en griego de los sustantivos sin artículo). Así, por ejemplo, (1) afirmaría que cuando pensamos en un hombre particular, Sócrates, pensamos en alguna de las cosas que son. (b) De acuerdo con una segunda interpretación, "ૂ $v \vartheta \rho \omega \pi o \varsigma$, por ejemplo, sería un término general usado para designar un universal, la propiedad de ser hombre. Así, (1) sostendría que, cuando pensamos en la propiedad de ser hombre, pensamos en alguna de las cosas que son.

Estas diferentes lecturas de (1) producen interpretaciones distintas del argumento. Sin embargo, ésta no es la única fuente de ambigüedad con respecto a cuál es el objeto del pensamiento en este argumento. De acuerdo con (1), cuando pensamos en $x$ pensamos en algo que es. Pero hay dos maneras distintas de interpretar esta afirmación. (i) Cuando pensamos en $x$ pensamos en algo que es, $y$, e $y$ es diferente de $x$. (ii) Cuando pensamos en $x$ pensamos en algo que es, $x$. Ambas lecturas son permisibles, pero no son combinables con cualquiera de las interpretaciones (a) y (b) ya expuestas. La interpretación (a), que $x$ es algún individuo particular, sólo es compatible con (i), pues, de acuerdo con la premisa (3), aquello que es, y en lo cual pensamos, no es alguna de las cosas particulares. Por tanto, (ii) queda descartada porque esta lectura identifica a $x$ con aquello que es, y $x$ según (a) es una cosa par-

${ }^{15}$ Cfr., por ejemplo, la primera premisa del segundo argumento de las ciencias: "de aquellas cosas que las ciencias son, estas cosas existen", 79. 8-9. Cherniss (1944, p. 272) llega incluso a identificar, erróneamente a mi juicio, el argumento del pensamiento con el segundo argumento de las ciencias.

${ }^{16}$ Fine (1993, p. 121) discute acertadamente esta cuestión. 
ticular, lo cual contradice a (3). De este modo, (a) sólo es compatible con (i), y de acuerdo con ambas lecturas, el argumento afirmaría que es posible pensar en cosas particulares sólo indirectamente, a través de pensamientos acerca de cosas que no son particulares. Por su parte, la interpretación (b), que $x$ es una propiedad, es consistente con (i) y (ii), pero creo que (ii) debe ser preferida. Con (b) y (i), el argumento afirmaría que cuando pensamos en una propiedad $F$, lo hacemos pensando en otra propiedad $G$, la cual es, y $G$ es una Idea. No obstante, aunque esta interpretación es plausible, creo que debe ser dejada en segundo plano, pues, según los ejemplos de (1) (hombre, pedestre, animal), debería concluirse que el argumento no identifica a hombre con la Idea de hombre, o bien, si lo hace, sostendría entonces que no podemos pensar directamente en la Idea de hombre, sino sólo a través de otra Idea. Ambas opciones parecen poco plausibles. Con (b) y (ii), el argumento afirmaría que cuando pensamos en una propiedad $F, F$ es, pensamos directamente en $F$, y $F$ es una Idea. Pienso que esta interpretación da una lectura más natural y razonable del argumento y, por tanto, me parece que (b) debe leerse con (ii). ${ }^{17}$

Ahora bien, ¿̇cuál de estas dos posibles lecturas de (1) y, a partir de ésta, del argumento en general, es la más adecuada? Me parece que (b) debe ser preferida. La razón principal es que, como veremos, el argumento es válido sólo si se interpreta de acuerdo con (b). Y, siguiendo un principio de caridad interpretativa, siempre es preferible no atribuir a un autor un argumento claramente inválido, si hay alguna otra opción interpretativa que sea plausible y según la cual el argumento sea válido. Esta interpretación difiere de la manera como usualmente se lee el argumento, pues algunos autores parecen pensar que éste intenta mostrar dos cosas: la existencia de universales objetivos y que éstos están separados. ${ }^{18}$ Sin embargo, de acuerdo con (b), en (1) parece suponerse que los universales son, pues (1) afirma que, cuando pensamos en una propiedad $F, F$ es. Así, según mi interpretación, el argumento no intenta mostrar la existencia de universales, sino que sostiene que, si

${ }^{17}$ Fine (1993, pp. 122-124) examina las posibles lecturas de (1), pero adopta una interpretación distinta de la que voy a proponer.

${ }^{18}$ Así parecen asumir Fine 1993, pp. 122-123, y Caston 1998, pp. 264-265. Aunque sus interpretaciones difieren entre sí, Fine piensa que el argumento es inválido para postular Ideas, pero válido para postular universales aristotélicos. Caston sostiene que el argumento es válido para postular Ideas, pero su interpretación es distinta de la mía. Cherniss (1944, p. 273) lee (1) de acuerdo con (b), pero su interpretación del argumento es muy diferente de la que propongo. Sobre esta cuestión véase también lo dicho en la n. 8. 
suponemos que existen tales universales (supuesto controvertido, pero que sería aceptado por Aristóteles), debemos concluir que éstos están separados.

De acuerdo con (1), cuando pensamos en hombre, pedestre o animal, pensamos en alguna de las cosas que son. Hay un aspecto más en que esta afirmación es todavía ambigua. El verbo "ser" en griego (Eival) es ambiguo porque un uso absoluto (no copulativo) del verbo puede tener sentidos diferentes: un sentido existencial, uno predicativo o uno veritativo. ${ }^{19}$ Así, la frase "las cosas que son" puede interpretarse, según el sentido existencial del verbo, como "las cosas que existen"; según el sentido predicativo, como "las cosas que son F"; y, según el veritativo, como "las cosas que son verdaderas". Pienso que عiv inal debe leerse existencialmente no sólo en (1), sino en todo el argumento. ${ }^{20}$ No obstante, creo que, si bien la lectura veritativa es claramente inadecuada, pues ésta requeriría que el objeto del pensamiento fuera una proposición, y además, que sólo fuera posible pensar proposiciones verdaderas, las lecturas existencial y predicativa son ambas prima facie plausibles. ${ }^{21}$ Victor Caston ha propuesto una lectura predicativa, argumentando que: (i) una lectura existencial del argumento hace que éste sea claramente inválido, mientras que (ii) una lectura predicativa lo hace válido, pero, (iii) por un principio de caridad interpretativa, debemos preferir la lectura predicativa. ${ }^{22} \mathrm{El}$ argumento de Caston es válido, de hecho, (ii) es verdadera, pues me parece que su interpretación predicativa hace que el argumento del pensamiento sea válido. Sin embargo, creo que no es un argumento sólido, pues (i) es falsa. Caston considera sólo la interpretación existencial de Gail Fine, la cual hace que el argumento sea claramente inválido, aunque Fine piense lo contrario (más adelante

${ }^{19}$ Cfr. Kahn 1966.

${ }^{20}$ Excepto en (5), donde es usado de acuerdo con un cuarto sentido, el de identidad.

${ }^{21}$ Fine (1993, pp. 121-122) defiende una interpretación existencial, pero las razones que da para apoyarla y rechazar una predicativa me parecen poco convincentes.

22 "El argumento, no obstante, puede fácilmente hacerse válido, si interpretamos la primera premisa como sigue: siempre que alguien piensa en una $F$, está en la relación pertinente, $T$, con respecto a algo que es ella misma $F[\ldots]$. Pero, entonces, la conclusión deseada se sigue claramente. Con respecto a cualquier sujeto $S$ y cualquier tipo $F, 1$. Siempre que $S$ piensa en $F, S$ está en $T$ con respecto a algo que es $F$. 2. $S$ piensa en $F$ de la misma manera incluso cuando no hay cosas particulares que son $F . \therefore 3$. Hay algo que es $F$ incluso cuando no hay cosas particulares que son F." (Caston 1998, p. 265).

Diánoia, vol. LIII, no. 60 (mayo 2008). 
diré por qué hace que sea inválido). ${ }^{23}$ Pero la interpretación de Fine no es la única posible. Mi interpretación ha utilizado, aunque no explícitamente, una lectura existencial (cuando sostengo que (1) supone la existencia de universales) y, no obstante, espero mostrar que mi interpretación hace, razonablemente, que el argumento sea válido. Por tanto, el argumento de Caston en contra de una lectura existencial carece de fuerza contra mi interpretación. Entonces, mi lectura existencial y la lectura predicativa de Caston están parejas en cuanto a este criterio de decisión. Pero creo que hay una razón para preferir una lectura existencial sobre una predicativa. La razón es que una lectura predicativa sugiere que tener propiedades tiene cierta prioridad ontológica sobre existir, para un objeto de pensamiento. Y esta tesis parece ser contraria a lo que Platón sostiene en al menos un par de pasajes de su obra, en República V (478b-c) y en Teeteto (189a-b), donde se afirma que no es posible opinar sobre lo que no es, afirmación que debe interpretarse existencialmente, pues es obviamente verdadero que sí se puede opinar acerca de lo que no es $F$, y también acerca de lo que no es verdadero, y en cambio, no es tan obvio que se pueda opinar o pensar en lo que no existe. Por estas razones creo que el argumento debe leerse de acuerdo con una interpretación existencial (en el sentido de existencia extramental).

De este modo, según las lecturas que he propuesto, (1) sostiene que cuando pensamos, por ejemplo, en caballo (la propiedad de ser caballo), pensamos en algo que existe. (2) Agrega que "el mismo pensamiento permanece incluso cuando éstas [las cosas particulares] se han destruido". Es decir, el mismo pensamiento acerca de la propiedad de ser caballo subsistiría incluso cuando no hubiera caballos particulares porque éstos se hayan destruido. Aunque (2) y (3) hablan en general acerca de "cosas particulares", debe entenderse que la extensión de este término incluye sólo a los miembros de la clase de particulares ejemplificada en el argumento, en este caso, la de los caballos. De otra manera, sería trivialmente falso que sería posible pensar en caballo incluso cuando las cosas particulares en su totalidad se hubieran destruido, pues no habría pensadores particulares. ${ }^{24}$ Asimismo, (2) describe la situación

${ }^{23}$ Cfr. Fine 1993, p. 129. Fine piensa que el argumento es inválido para postular Ideas, pero válido para postular universales aristotélicos. Sin embargo, la objeción contra Fine es que su interpretación existencial hace que éste sea claramente inválido para postular no sólo Ideas, sino universales de cualquier índole.

${ }^{24}$ En esta restricción de (2) a una clase determinada de particulares, sigo a Caston 1998, pp. 264-265. También lo sigo en el uso de un ejemplo distinto (caballo) de los utilizados en el argumento, hombre, pedestre y animal, para simplificar la 
que se presentaría cuando todos y cada uno de los miembros de una clase determinada, la de los caballos en este caso, se hubieran extinguido. Esto es, (2) no afirma que el mismo pensamiento permanece cuando cualquier individuo dado, miembro de la clase pertinente (pero no necesariamente todos), se ha destruido, ${ }^{25}$ pues el plural "incluso

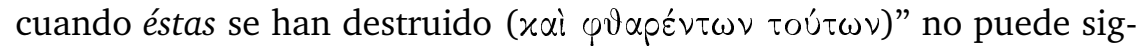
nificar esto.

Pero si esta interpretación es correcta, entonces el argumento concluye válidamente la existencia de universales separados, y, por consiguiente, de Ideas, pues, de acuerdo con (2), podemos pensar en la propiedad de ser caballo aun cuando todos los caballos particulares hayan dejado de existir; pero, por (1), este objeto del pensamiento es algo que existe, por tanto, (4) la propiedad de ser caballo puede existir aunque no haya caballos particulares que la instancien. Y esto es precisamente lo que debe entenderse por la separación de las Ideas, rasgo distintivo de los universales platónicos. En efecto, la separación es una característica modal de las Ideas, la cual consiste en la capacidad que éstas tienen de existir sin ser instanciadas. Las Ideas son ontológicamente independientes de las cosas que las instancian. ${ }^{26}$ (5) Hace explícita esta identificación de universales separados con Ideas y sugiere que, según el argumento, concluir la separación de los universales es una condición suficiente para concluir la existencia de Ideas, conclusión que he hecho explícita en (6), pero que en el texto está omitida.

De acuerdo con esto, la función de (3), que cuando pensamos en caballo no pensamos en ninguno de los caballos particulares, no es tan importante para el argumento, pues (3) explicita algo que ya se había supuesto en (1), que cuando pensamos en caballo pensamos en la propiedad de ser caballo. Este supuesto no es obvio, pero no debe interpretarse que (3) agrega al argumento algo que no estaba ya contenido en éste, en especial no debe interpretarse que (3) proporciona la razón por la cual se concluye en (4) que existen los universales, pues, si así fuera, el argumento sería inválido, como mostraré a continuación.

explicación, pues siendo el tercero el género al que pertenecen los seres humanos, y el segundo una de sus diferencias específicas, el argumento sostendría que el pensamiento acerca de hombre permanece incluso cuando no hay hombres particulares, y surgiría la cuestión (complicación evitable) de qué sujeto no humano podría pensar este pensamiento (¿una divinidad?).

${ }^{25}$ Interpretación que Fine (1993, pp. 124, 128-129, 312 n. 28) parece asumir. Caston (1995, p. 165) y (1998, p. 264) critica acertadamente esta interpretación.

${ }^{26}$ Véase la n. 3.

Diánoia, vol. LIII, no. 60 (mayo 2008). 
He mencionado que otro tipo de versión existencial del argumento, como la de Gail Fine, ocasiona que éste sea inválido para concluir no sólo la existencia de Ideas, sino la existencia de universales de cualquier índole. Esta interpretación asume la lectura (a) ya expuesta, según la cual (1) no implica un compromiso ontológico con la existencia de universales, sino que identifica al objeto del pensamiento con una cosa particular cualquiera. Después esta versión sostiene que, según (2), podemos pensar en caballo aunque no haya caballos particulares, y que de (2) se sigue (3), crucial para esta interpretación, que cuando pensamos en un caballo no pensamos en ninguno de los caballos particulares, subconclusión que añade algo nuevo que no estaba ya contenido en el argumento. Después esta interpretación afirma que (4) intenta concluir, a partir de (1-3): (i) que existen los universales y (ii) que éstos están separados.

El problema con esta interpretación es que (4.i) no se sigue válidamente de (1-3), pues de estas premisas sólo se sigue que existe algo en lo cual pensamos cuando pensamos en caballo, aun cuando los caballos particulares se hayan destruido, y que este algo no es idéntico a ninguno de éstos. Pero el argumento deja abierta la posibilidad de que este objeto del pensamiento sea cualquier cosa particular siempre y cuando ésta no sea un caballo. Así pues, el argumento, interpretado de esta manera, no da razones suficientes para garantizar la verdad de la conclusión de que este objeto del pensamiento es un universal y, por tanto, (4.i) no es una conclusión válida. Y, obviamente, tampoco es válida (4.ii). Parece entonces imposible concluir válidamente la existencia de los universales a partir de (a) y de una lectura existencial. ${ }^{27}$ Pero esto no representa ningún problema para la interpretación que defiendo, porque ésta sostiene que no es uno de los objetivos del argumento concluir la existencia de universales, sino que presuponiendo ésta se pretende concluir su separación.

Así, de acuerdo con la interpretación que he propuesto, el argumento del objeto del pensamiento puede formularse más precisamente del siguiente modo (incluyo en corchetes angulares una simbolización aproximada de cada premisa):

$(1)^{*}$ Necesariamente (Si pensamos en la propiedad de $\operatorname{ser} F$, entonces, la propiedad de ser $F$ existe). $\langle\square[P a F \rightarrow(\exists X)(X=F)]\rangle$

${ }^{27}$ Contrariamente a lo que Fine (1993, pp. 128-129) sostiene, seguida por Santa Cruz, Crespo y Di Camillo (2000, p. 32). 
$(2)^{*}$ Es posible (Pensar en la propiedad de ser $F$ y que no existan cosas particulares que son $F) .\langle\diamond[P a F \& \neg(\exists x) F x]\rangle$

$(3)^{*}$ Es posible (Que la propiedad de ser $F$ exista y que no existan cosas particulares que son $F) .\langle\diamond[(\exists X)(X=F) \& \neg(\exists x) F x]\rangle$

$(4)^{*}$ La propiedad de ser $F$ es una Idea. $\langle I(F)\rangle$

$(5)^{*}$ Existen las Ideas. $\langle(\exists X)[(X=F) \& I(X)]\rangle^{28}$

Espero que esta formulación más rigurosa del argumento permita apreciar más claramente su forma lógica y, por ende, su validez. De acuerdo con esta formulación, (1)* es una especie de principio (por esta razón debe ser considerado necesario, como lo he hecho explícito) que establece que, necesariamente, si pensamos en una propiedad $F$ cualquiera, esa propiedad existe (véase más sobre este principio en la sección 5). (2)* Afirma la posibilidad de, conjuntamente, pensar en la propiedad de ser $F$ y que no haya individuos que tienen esa propiedad de ser $F$. Mientras que (3)* es una subconclusión que afirma la posibilidad de que, conjuntamente, exista la propiedad de ser $F$ y no haya individuos que sean $F$. Esto es, (3)* afirma la separación de la propiedad de ser $F$, pues la separación consiste precisamente en esta característica modal. Y $(3)^{*}$ se sigue válidamente de $(1)^{*}$ y $(2)^{*}$, porque ejemplifica una forma de inferencia que es válida en lógica modal proposicional: 1 . $\square(p \rightarrow q)$, 2. $\diamond(p \& r), 3 . \diamond(q \& r)$. (4)* y (5)* derivan explícitamente la conclusión de que existen las Ideas, la cual se sigue de la subconclusión que afirma válidamente la separación de los universales (junto con dos premisas auxiliares que en este caso, por afán de simplificación, he omitido: ( $\alpha$ ) PaF y $(\beta)(\exists X)(X=F)$, donde $(\beta)$ se sigue, por modus ponens, de $\left.(1)^{*} \mathrm{y}(\alpha)\right) .^{29}$

${ }^{28}$ Aclaración sobre los símbolos lógicos usados: ‘ $\square$ ’ y ‘ $\diamond$ ’ son los operadores modales de necesidad y posibilidad, respectivamente; la variable ' $X$ ' es de segundo grado, tiene como valores sólo propiedades y relaciones, mientras que la variable ' $x$ ' es de primer grado, tiene como valores sólo a individuos. Me parece que el resto de los símbolos usados no requiere mayor explicación.

${ }^{29} \mathrm{El}$ argumento del objeto del pensamiento, tal y como está formulado en el Peri ideōn, parece afirmar que, a partir de la consideración de que podemos pensar en un universal $U$ en $t_{2}$ cuando las cosas particulares que lo instanciaban en $t_{1}$ ya no existen en $t_{2}$, se sigue que $U$ existe en $t_{2}$, y que, por tanto, está separado de las cosas que lo instancian (es una Idea). Sin embargo, según David Armstrong, ésta no es una condición suficiente para afirmar la separación y, por tanto, la existencia de universales platónicos. Armstrong acepta un realismo de universales, que él denomina "aristotélico", y defiende un principio de instanciación, según el cual un universal existe si y sólo si es instanciado en al menos un tiempo $t$ : "el cuantificador 
De este modo, hemos visto que uno de los argumentos "menos rigurosos" del Peri ideōn, el del objeto del pensamiento, es válido para postular Ideas. Esto parece indicar que la manera usual de interpretar la distinción entre argumentos "menos y más rigurosos" es errónea, pues, según ésta, los argumentos "menos rigurosos" lo son porque son inválidos para postular Ideas. Sin embargo, dado que es Aristóteles quien establece la distinción en cuanto rigor en Metafísica A 9, la interpretación tradicional podría ser defendida argumentando que quizá Aristóteles mismo consideraba que el argumento del objeto del pensamiento era inválido y que, por tanto, aun si la interpretación del argumento que he propuesto es correcta, tan sólo podría concluirse de ella que Aristóteles estaba equivocado, pero no que la distinción entre argumentos más y menos rigurosos no obedece a una distinción en cuanto a su validez. Me parece que esta defensa es inadecuada. A continuación analizaré las críticas que Aristóteles dirige al argumento del objeto del pensamiento, pues éstas son la única evidencia textual que tenemos para juzgar este asunto. Me parece que las críticas de Aristóteles sugieren que él consideraba que el argumento es válido pero no sólido. En la próxima sección discutiré las críticas de Aristóteles de manera general y estudiaré la primera crítica explícita de Aristóteles. Después, en la sección 5 me ocuparé de la segunda crítica.

existencial nada tiene que ver con el momento actual. Que $(\exists x)$ (Brontosauro $x$ ) es verdadera aun cuando, presumiblemente, $(\exists x)$ (Unicornio $x$ ) es falsa. Un universal existe si hubo, hay o habrá particulares que tengan esa propiedad o que se encuentren en esa relación" (1978, p. 188). Es evidente que el caso presentado en la premisa (2) del argumento del objeto del pensamiento no viola este principio. Armstrong identifica a los universales platónicos como universales que pueden existir sin ser instanciados en ningún tiempo $t$. Creo que Armstrong tiene razón en parte, esta posibilidad de absoluta no instanciación es la versión más fuerte y característica del realismo platónico de universales. No obstante, pienso que para Platón ambos tipos de no instanciación, momentánea y absoluta, serían para él casos de separación, pues sospecho que no encontraría una diferencia esencial entre una y otra. El asunto central es que, para Platón, la separación de los universales consiste en la independencia ontológica que éstos tienen con respecto a las cosas particulares: un universal puede existir aunque no haya ninguna cosa particular que lo instancie. Y esta independencia incluye tanto casos en que un universal es instanciado en algún tiempo $t_{1}$, pero no lo es en un tiempo $t_{2}$, como casos en los que no es instanciado en ningún tiempo $t$. De cualquier modo, el argumento del objeto del pensamiento es válido primordialmente para concluir la no instanciación absoluta (como puede verse en la formalización propuesta). Como veremos más adelante, la segunda crítica de Aristóteles ejemplifica esto, pues, según esa crítica, el argumento sería válido para postular una Idea de hipocentauro, aunque nunca ha habido ni habrá hipocentauros. 
4. La primera crítica de Aristóteles: ¿Ideas de individuos?

Como he mencionado, Aristóteles dirige explícitamente dos críticas al argumento del objeto del pensamiento: que de éste se seguirían (i) Ideas correspondientes a cada individuo, y (ii) Ideas de seres ficticios. Me parece que ambas críticas están encaminadas a cuestionar la solidez del argumento. De hecho, no parece haber ningún indicio fidedigno de que Aristóteles dudara de la validez de este argumento. Es cierto que, si bien la versión más confiable del Peri ideōn (OAC) no contiene una crítica explícita a la validez del argumento, tal crítica está presente en la versión alterna, menos confiable, (LF). ${ }^{30}$ Según (LF), el argumento no es válido para postular Ideas, sino que sólo es válido para postular universales aristotélicos. Sin embargo, considero que (LF) no es una fuente adecuada en la cual pueda basarse una interpretación del Peri ideōn. Las importantes variaciones que presenta con respecto a (OAC) obligan a considerarlo como un texto secundario y de importancia muy menor. Además, me parece tendencioso pretender utilizarlo selectivamente, pues sólo se recurre a él cuando (OAC) no respalda la interpretación que se quiere defender. ${ }^{31}$

Podría sugerirse, quizá, que una crítica a la validez del argumento está indicada en la manera como Aristóteles concluye sus críticas a éste: "de modo que tampoco este argumento concluye que hay Ideas (oủoc̀

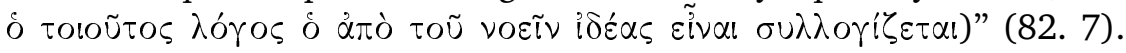
Esta hipótesis podría apoyarse, acaso, en el hecho de que en las críticas de Aristóteles al argumento de lo uno sobre muchos (81. 8-10),

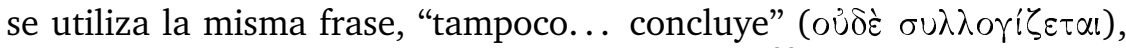
para cuestionar la validez de dicho argumento. ${ }^{32}$ Además, el término

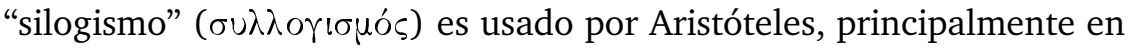
los Analíticos posteriores, para significar "deducción". Sin embargo, me parece que la frase por sí misma no contiene un cuestionamiento a la validez del argumento, pues en el de lo uno sobre muchos la crítica a la validez aparece sólo cuando se le añade otra frase en la que se afirma que el argumento serviría para mostrar sólo la existencia de entidades distintas de las particulares, pero no necesariamente la existencia de Ideas. Esta segunda frase está ausente en (OAC) en las críticas al argumento del objeto del pensamiento. Pienso que ante tal ausencia sólo

${ }^{30}$ Sobre estos diferentes textos, véase la n. 11.

${ }^{31}$ Como hace Fine 1993, pp. 120, 128-129.

${ }^{32}$ Asimismo, en la crítica que aparece en el texto espurio (LF) se utilizan las mismas palabras, pero se añade después la crítica explícita a la validez del argumento (82. 7-9).

Diánoia, vol. LIII, no. 60 (mayo 2008). 
estamos legitimados a interpretar la frase con base en las críticas que la preceden; esto es, los indicios textuales proporcionados por (OAC) indican que la frase es una recapitulación de lo que las críticas anteriores han logrado a juicio de Aristóteles: cuestionar la solidez del argumento y, por tanto, su uso para concluir la existencia de Ideas. Asimismo, si

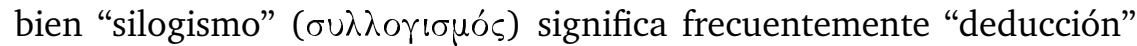
en las obras de Aristóteles, en algunos lugares el verbo $\sigma \cup \lambda \lambda o \gamma$ i $\varepsilon \sigma \vartheta \alpha l$ significa simplemente "concluir" o incluso "recapitular" (como en $\mathrm{Me}$ tafísica H 1. 1042a3).

En cualquier caso, la mejor guía que poseemos es la interpretación misma del argumento, y espero haber dado razones suficientes para pensar que mi interpretación es preferible (y más plausible) a la estándar, que hace al argumento inválido, tanto para postular Ideas como para postular universales de cualquier índole, pues, entre otras cosas, cumple razonablemente con el principio de caridad interpretativa de no atribuir a un autor un razonamiento claramente inválido cuando hay alguna otra opción plausible en la que éste es válido. Como hemos visto, la interpretación tradicional le atribuiría un defecto claro de validez al argumento platónico. Y, además, la interpretación tradicional atribuiría un segundo error grave, esta vez a Aristóteles, al adjudicarle una crítica a la validez del argumento según la cual éste no es válido para postular Ideas, pero sí lo es para postular universales aristotélicos, pues, como espero haber mostrado en la sección anterior, si el argumento es acaso válido, lo es sólo para postular universales platónicos, pero de ninguna manera para postular universales aristotélicos o de cualquier otra índole. Así, por las razones anteriores, me parece plausible concluir que no hay en las críticas de Aristóteles al argumento un cuestionamiento a su validez. Como veremos, las dos críticas explícitas que Aristóteles elabora tienen por objetivo cuestionar la solidez del argumento. En esta sección analizo la primera crítica y en la siguiente la segunda. Primero, dado que Aristóteles sostiene que el argumento no es sólido, voy a comentar brevemente sobre cuál pienso que es la premisa de este argumento que él rechaza. Después voy a evaluar la primera crítica de Aristóteles. Considero que esta crítica es justa y que le plantea al argumento un problema grave que hace dudar de su solidez. Me parece que ninguna de las posibles salidas platónicas a esta crítica es satisfactoria.

Según la interpretación que he propuesto, el argumento del objeto del pensamiento es válido para postular Ideas. Ahora bien, ¿es este argumento, además de válido, sólido, esto es, tiene premisas verdaderas? Aristóteles presenta dos críticas al argumento que componen una 
respuesta negativa a esta pregunta. La objeción de Aristóteles es que del argumento se siguen válidamente más universales platónicos de los que sería razonable postular. Las dificultades que Aristóteles detecta en el argumento parecen ser causadas por (1): "si pensamos en hombre, pedestre o animal, pensamos en alguna de las cosas que son". Sugiero que ésta es la premisa que Aristóteles rechaza y de la cual se siguen las conclusiones problemáticas que Aristóteles detecta en sus críticas. No voy a examinar, por el momento, de manera directa y aislada si (1) es razonable, sino que voy a analizar la primera crítica de Aristóteles, y después la segunda, de esta manera se apreciarán más claramente las dificultades que (1) suscita.

La primera crítica aristotélica sostiene que "este argumento establece Ideas de las cosas que se destruyen y de las que están destruidas y, en general, de las cosas particulares y destructibles, como Sócrates o Platón" (82. 1-3). La crítica consiste en que, según Aristóteles, el argumento es válido para postular una Idea correspondiente a cada individuo particular (Sócrates, Platón, etcétera) y no sólo una Idea común a los miembros de cierta clase de cosas (una Idea correspondiente a los miembros de la clase de los hombres, una correspondiente a los miembros de la clase de los caballos, etcétera). Las razones que Aristóteles da para apoyar esta objeción son: "en efecto, también pensamos en estas cosas y retenemos una imagen de éstas, incluso cuando ya no son" (82. 4-5). De acuerdo con la interpretación propuesta en la sección anterior, la crítica de Aristóteles requiere ser interpretada, para ser válida, suponiendo que el objeto del pensamiento al que se alude es un universal (en este caso, la propiedad de ser Sócrates) y no un particular (Sócrates mismo). A continuación presento una formulación de esta primera crítica, que sigue a la presentación más rigurosa del argumento propuesta al final de la sección anterior (omito, para simplificar, la simbolización lógica):

(1) Necesariamente (Si pensamos en la propiedad de ser Sócrates, entonces, la propiedad de ser Sócrates existe).

(2) Es posible (Pensar en la propiedad de ser Sócrates y que Sócrates no exista).

(3) Es posible (Que la propiedad de ser Sócrates exista y que Sócrates no exista).

(4) La propiedad de ser Sócrates es una idea.

(5) Existe la Idea de Sócrates. 
Este argumento es válido. La clave está, como he señalado antes, en que (1) supone la existencia de la propiedad de ser Sócrates, de modo que el argumento sólo intenta dar razones a favor de la separación de ésta con respecto a Sócrates. La inferencia de la separación es válida, pues, según (1), cuando pensamos en la propiedad de ser Sócrates esta propiedad existe y, según (2), es posible pensar en esta propiedad incluso cuando Sócrates ya no existe, por tanto, la propiedad de ser Sócrates puede existir aunque Sócrates no exista. Como he mencionado, este razonamiento ejemplifica una forma de inferencia válida en lógica modal proposicional: $1 . \square(p \rightarrow q), 2 . \diamond(p \& r), \therefore 3 . \diamond(q \& r)$. Así, la crítica de Aristóteles concluye válidamente que la propiedad de ser Sócrates es una Idea en virtud de que ésta es ontológicamente independiente de Sócrates. De este modo, dado que la primera crítica de Aristóteles es correcta, es preciso examinar si ésta detecta en verdad un problema importante para el realismo platónico de universales y si Platón tiene alguna salida razonable a esta dificultad.

La respuesta platónica más inmediata a esta crítica sería, por supuesto, aceptar la existencia de Ideas de individuos, manteniendo que no surge ninguna dificultad por admitir estas Ideas. Algunos filósofos, como Leibniz y Plantinga, han postulado este tipo de propiedades de individuos. ${ }^{33}$ A este tipo de propiedades se les ha llamado "ecceidades". ${ }^{34}$ Una ecceidad se define como una propiedad que es necesaria para un individuo $x$ ( $\sin$ la cual $x$ no podría existir) y que, además, es imposible que una cosa distinta a $x$ la instancie. Una ecceidad es, pues, una suerte de esencia individual que identifica, necesariamente, de manera única a uno y sólo un individuo. Sin embargo, pienso que Platón no podría aceptar este tipo de Ideas, pues, para Platón, las Ideas son entidades que se postulan para explicar la naturaleza común a varias cosas y no la naturaleza estrictamente individual de una cosa particular. En efecto, las Ideas son universales, y una de las condiciones necesarias para ser un universal es la de ser instanciable por múltiples, quizá infinitas, cosas. ${ }^{35}$ No obstante, las ecceidades sólo pueden ser instanciadas por uno y sólo un objeto; por tanto, las ecceidades no son, estrictamente, universales, sino lo que se ha llamado "tropos", propiedades particula-

${ }^{33}$ Cfr. Plantinga 1974, pp. 70-87. Al parecer, los estoicos antiguos tuvieron una noción semejante de propiedad individual o singularizada; véase, Salles y Molina 2004, pp. 175-177.

34 "Ecceidad" es la castellanización del término latino haecceitas, el cual proviene de haec, "esto", y significa literalmente "estidad". Duns Escoto acuñó este término, aunque con un uso distinto del leibniziano.

${ }^{35}$ Como sostiene acertadamente Armstrong 1978, p. 135. 
rizadas. ${ }^{36}$ Por ende, Platón no podría admitir la existencia de Ideas de esta índole, pero estaría comprometido a hacerlo si quisiera al mismo tiempo usar el argumento del objeto del pensamiento para postular válidamente la existencia de Ideas.

Las únicas dos estrategias que se me ocurre que Platón podría utilizar para evitar esta consecuencia problemática del argumento del objeto del pensamiento no son satisfactorias. Una sería sostener que en realidad no es posible pensar en la propiedad de ser Sócrates de manera adecuada como para poder concluir que ésta existe; es decir, que este tipo de propiedades son absurdas y que, por tanto, ni siquiera se pueden concebir. Sin embargo, no creo que esta estrategia sea apropiada, pues a muchos no les ha parecido que postular estas propiedades sea absurdo. $Y$ en verdad no parece haber nada claramente absurdo en ellas, siempre y cuando no se afirme que éstas son universales. Para rechazar estas propiedades se requerirían razones más sólidas y precisas, pero esto evidencia que éstas son enteramente concebibles.

Otra estrategia sería admitir que estas propiedades de individuos existen, pero que éstas no son Ideas (universales), sino tropos. Sin embargo, aunque esta tesis es razonable, el problema radica en que el argumento del objeto del pensamiento sostiene que cualquier propiedad pensable existe, está separada y es, por tanto, una Idea. Creo que éste es justamente el problema que la primera crítica aristotélica detecta con respecto a (1), y como consecuencia, con respecto a la solidez del argumento: que (1) pasa demasiado fácilmente de la posibilidad de pensar en una propiedad $F$ a la aceptación de la existencia de $F$ (la cual después es identificada como una Idea). Éste es un problema grave que amenaza seriamente la solidez del argumento platónico del pensamiento. En la próxima sección veremos que es este mismo problema el que suscita la segunda crítica aristotélica.

\section{La segunda crítica de Aristóteles: ¿Ideas de cosas ficticias?}

En esta sección analizo la segunda crítica de Aristóteles al argumento del objeto del pensamiento, que de éste se seguirían Ideas de cosas ficticias, la cual, me parece, tiene por finalidad cuestionar su solidez. Considero que esta crítica es justa pero que, en sí misma, no representaría un problema para un realismo platónico de universales. Sin embargo, pienso que esta crítica y la estudiada en la sección anterior indican que

\footnotetext{
${ }^{36}$ Sobre la diferencia entre tropos y universales véanse: Armstrong 1978,
} pp. 116-129, y Lewis 1986, pp. 63-69.

Diánoia, vol. LIII, no. 60 (mayo 2008). 
la premisa (1) es muy probablemente falsa y que, por tanto, el argumento no es sólido: la aceptación de la existencia de una propiedad $F$ a partir de la posibilidad de pensar en $F$ da lugar a la postulación de muchas Ideas problemáticas, además de las Ideas de individuos de la primera crítica.

Aristóteles expone escuetamente su segunda crítica al argumento afirmando que "también pensamos en las cosas que no son en absoluto, como en hipocentauro o Quimera" (82. 5-6). Pienso que, como en el caso de la primera crítica aristotélica, en esta segunda debe interpretarse, para que ésta sea válida, que el objeto del pensamiento es un universal y no un particular. Así, esta crítica puede formularse de la siguiente manera:

(1) Necesariamente (Si pensamos en la propiedad de ser hipocentauro, entonces, la propiedad de ser hipocentauro existe).

(2) Es posible (Pensar en la propiedad de ser hipocentauro y que no haya hipocentauros).

(3) Es posible (Que la propiedad de ser hipocentauro exista y que no haya hipocentauros).

(4) La propiedad de ser hipocentauro es una Idea.

(5) Existe la Idea de hipocentauro.

Este argumento es válido para concluir la separación de la propiedad de ser hipocentauro, pues, por (2), podemos pensar en esta propiedad aunque no haya hipocentauros; pero, por (1), si pensamos en la propiedad de ser hipocentauro, ésta existe; por tanto, esta propiedad puede existir aunque no haya hipocentauros. Como he mencionado antes, el argumento ejemplifica la forma de inferencia válida: $1 . \square(p \rightarrow q)$, 2. $\diamond(p \& r), 3 . \diamond(q \& r)$. De este modo, la propiedad de ser hipocentauro es ontológicamente independiente de los hipocentauros particulares que pueden instanciarla y, por ende, es una Idea. Un aspecto interesante de esta crítica es que ejemplifica que el argumento es válido para postular universales absolutamente no instanciados, universales que no son instanciados en ningún tiempo $t$; ya que nunca ha habido ni habrá hipocentauros.

Así, la crítica aristotélica es justa; si Platón pretende usar este argumento para postular Ideas, debe aceptar la existencia de Ideas de entidades ficticias como hipocentauros. Aristóteles piensa que esto es problemático; pero ¿en verdad surge alguna dificultad para un platónico si acepta este tipo de Ideas? Creo que la respuesta a esta pregunta es 
negativa. Habría un problema si el argumento llevara a la conclusión de que existen hipocentauros. Pero el argumento no establece esto, sino solamente que existe la propiedad de ser hipocentauro, haya o no haya cosas que la instancien. Sin embargo, esto es simplemente realismo platónico de universales, y no veo ningún problema obvio en aceptar esta consecuencia, sobre todo si se toma en consideración que es metafísicamente posible que haya hipocentauros y quimeras; es decir, que aunque en este mundo no existan monstruos de este tipo, si algún otro mundo posible hubiera sido real estos monstruos habrían existido. ${ }^{37}$ Así, podría proponerse que la Idea de hipocentauro, si bien no es instanciada en este mundo, es posiblemente instanciada, o instanciada en otros mundos posibles (en aquellos de acuerdo con los cuales hay hipocentauros). Y creo que una de las condiciones que debe cumplir un universal platónico es la de ser posiblemente instanciado. Por tanto, no me parece que la postulación de Ideas correspondientes a entidades ficticias le cause ningún problema a una teoría platónica de Ideas. ${ }^{38}$ La única objeción que la segunda crítica aristotélica le podría presentar al realismo platónico de universales sería una protesta de incredulidad ante la existencia de universales absolutamente no instanciados. Pero esta incredulidad no constituye en sí un argumento en contra de éstos.

Con todo, esta segunda crítica revela, lo mismo que la primera, un problema serio con el argumento del objeto del pensamiento. Este problema radica en que (1) autoriza a postular la existencia de cualquier universal que sea posible pensar, universal que el argumento identifica válidamente con una Idea. Aunque postular Ideas de entidades ficticias no sea problemático para Platón, hemos visto que postular Ideas de individuos sí lo sería. Pero el argumento no sólo sería válido para postular este tipo de Ideas, sino prácticamente cualquier Idea problemática que podamos pensar. Por ejemplo, éste sería válido para postular Ideas imposibles de instanciar, como la Idea de ser distinto de sí mismo o la de ser un círculo cuadrado, pues podemos pensar en distinto de sí mismo y en círculo cuadrado, a pesar de que es imposible que haya cosas distintas de sí mismas y círculos cuadrados. La postulación de estas Ideas violaría la condición que me parece que un platonismo cauto debería aceptar: que un universal existe si y sólo si es posiblemente instanciado.

${ }^{37}$ Con esto no pretendo afirmar que los hipocentauros de la mitología griega podrían haber existido, sino simplemente que entidades con las características que se les atribuye a éstos podrían haber existido. Éstas son dos tesis diferentes, como advierte Kripke (1980, pp. 24 y 156-158).

${ }^{38}$ Por supuesto, Platón bien pudo rechazar la existencia de Ideas de entidades ficticias por cualesquiera otras razones.

Diánoia, vol. LIII, no. 60 (mayo 2008). 
Se podría intentar bloquear esta consecuencia sosteniendo que el tipo de pensamiento del cual habla el argumento involucra un tipo de concebibilidad más riguroso. De este modo, podría argumentarse que es imposible concebir propiedades como la de ser distinto de sí mismo y la de ser un círculo cuadrado, y que, por tanto, del argumento no se seguiría la existencia de éstas. Sin embargo, aun concediendo esta interpretación (la cual me parece improbable), la objeción subsistiría, pues aunque parece plausible sostener que es imposible concebir, con detalle, un objeto imposible, creo que sí es posible concebir hasta cierto punto propiedades imposibles de instanciar. ${ }^{39}$ La diferencia radica en que cuando concebimos una de estas propiedades no concebimos una contradicción, como sí ocurriría si fuera posible concebir que un objeto instancia estas propiedades. Cuando concebimos la propiedad de ser un círculo cuadrado, concebimos las condiciones que se requieren para que $x$ sea un círculo cuadrado, y éstas son que $x$ sea circular y cuadrado al mismo tiempo y en toda su superficie. Pero en este caso no concebimos una contradicción, lo cual ocurriría si concibiéramos el objeto imposible el círculo-cuadrado, sino las condiciones para que se dé tal contradicción. ${ }^{40}$ Así pues, el argumento del objeto del pensamiento justificaría la postulación de Ideas imposibles de instanciar. Pero también se seguirían de éste muchas otras Ideas problemáticas, algunas de las cuales son advertidas por Aristóteles como consecuencias inaceptables de otros argumentos del Peri ideōn: las Ideas de artefactos de los argumentos de las ciencias, las Ideas de negaciones del argumento de lo uno sobre muchos, las Ideas de términos relativos del argumento de los relativos, y la Idea del tercer hombre del argumento que lleva este título, pues podemos pensar en cama, no-caballo, etc. El argumento también sería válido para postular Ideas disyuntivas, como la Idea de ser blanco $o$ redondo, pues podemos pensar en blanco o redondo, e Ideas arbitra-

${ }^{39}$ Digo "hasta cierto punto" porque tengo en mente un tipo de concebibilidad que no garantiza posibilidad. Mi tesis es simplemente que aun interpretando el argumento de acuerdo con un concepto de "pensamiento" un poco más fuerte, la objeción subsistiría porque, si bien no es posible concebir en ningún sentido un objeto imposible, sí es posible concebir, en algún sentido, una propiedad imposible de instanciar. Esto llevaría al argumento a postular, por ejemplo, una Idea de círculocuadrado, a pesar de que, en última instancia, la concebibilidad en cuestión no sería suficiente para concluir la posibilidad, pues se trataría de cierta concebibilidad débil que exige sólo que no sea contradictorio pensar una situación dada, pero esto no garantiza que la situación sea posible.

${ }^{40}$ Esta tesis está basada en el análisis fregeano del concepto "no idéntico a sí mismo" (los conceptos fregeanos son parientes cercanos de las Ideas platónicas); cfr. Frege 1954, párrafo 74. 
riamente generadas como la Idea de ser verzul, verde hasta $t_{1} \mathrm{y}$ azul a partir de $t_{2}$, pues podemos pensar en verzul. En general, el argumento es válido para postular cualquier universal problemático que podamos pensar.

Como he mencionado, el problema con el argumento radica en la premisa (1), pues ésta impone la cuestionable condición de que para poder pensar en la propiedad $F, F$ debe existir. Sin embargo, una condición más razonable sería que para poder pensar en la propiedad $F$, nuestro pensamiento debe tener algún contenido, pero esto no implica que $F$ exista. Este contenido puede ser una proposición, una representación mental o alguna otra entidad adecuada que pueda servir como objeto intencional, objeto hacia el cual está dirigido nuestro pensamiento cuando pensamos en $F$. Así pues, este argumento parece cometer el error de confundir entre objeto y contenido de pensamiento, distinción que, por cierto, la desarrolla Aristóteles en De Anima (especialmente en 3. 4-8). ${ }^{41}$ Es preciso señalar, no obstante, que la condición impuesta en (1) no está necesariamente vinculada con un realismo platónico de universales, ésta es cuestionable en sí misma y resulta problemática para cualquier realismo de universales, pues es inaceptable sostener que hay un universal objetivo por cada propiedad que podamos pensar. Esto lleva a la postulación de más universales de los que es razonable aceptar. $^{42}$

\section{Conclusión}

Hemos visto que uno de los argumentos "menos rigurosos" del Peri ideōn, el argumento del objeto del pensamiento, es válido para postular Ideas, pues es válido para postular universales separados, y la separación es uno de los rasgos distintivos de las Ideas. Hemos visto que las únicas críticas confiables que Aristóteles formula contra este argumento tienen como objetivo cuestionar su solidez, pero no su vali-

${ }^{41}$ Tal distinción parece estar sugerida en la primera crítica aristotélica, cuando después de afirmar que podemos pensar en Sócrates o Platón sostiene que "también pensamos en estas cosas y retenemos una imagen de éstas, incluso cuando ya no son" (82. 3-4). Caston (1998) ofrece una explicación de la teoría aristotélica del pensamiento. Según Anscombe (1993, pp. 97-98), la teoría de las Ideas de Platón tiene su origen en un error semejante, en pasar de reconocer la existencia intencional de significados y conceptos, a afirmar la existencia extramental de éstos como Ideas.

${ }^{42}$ El argumento del objeto del pensamiento no es sólido; sin embargo, parece tener auténtica filiación platónica. Los pasajes ya mencionados de República V 478b-c y Teeteto 189a-b presentan razonamientos muy semejantes a este argumento.

Diánoia, vol. LIII, no. 60 (mayo 2008). 
dez. Esto, me parece, es un buen indicio para sostener que Aristóteles concuerda con la interpretación del argumento que he presentado. Pero si esta interpretación es correcta, entonces es preciso rechazar la interpretación estándar de la diferencia entre argumentos "más y menos rigurosos" del Peri ideōn, según la cual los argumentos "menos rigurosos" lo son porque no son válidos para postular Ideas, mientras que los "más rigurosos" sí lo son. No he intentado desarrollar en este trabajo una interpretación alternativa de la distinción en cuanto a rigor que se establece en Metafísica A 9. Mi propuesta consiste más bien en cuestionar el papel primordial que se le ha adjudicado a dicha distinción en la interpretación del Peri ideōn. Me parece que, si no se puede establecer una distinción entre el grupo de los argumentos "menos rigurosos" y el de los "más rigurosos" en cuanto a validez o en cuanto a solidez, entonces la distinción no es realmente tan importante como se ha creído y no debe, por tanto, constituir el eje central de interpretación del Peri ideōn. ${ }^{43}$

\section{BIBLIOGRAFÍA}

Anscombe, G.E.M., 1993, “The Origin of Plato's Theory of Forms”, en R.W. Sharples, Modern Thinkers and Ancient Thinkers, University College London Press, Londres, pp. 90-99.

Armstrong, D.M., 1988, Los universales y el realismo científico, trad. J.A. Robles, Instituto de Investigaciones Filosóficas-UNAM, México; 1a. ed.: Universals and Scientific Realism, Cambridge University Press, Cambridge, 1978.

Caston, V., 1998, "Aristotle and the Problem of Intentionality", Philosophy and Phenomenological Research, vol. 58, no. 2, pp. 249-297.

—, 1995, "Review of Gail Fine: On Ideas", Mind, vol. 104, pp. 162-166.

Cherniss, H., 1944, Aristotle's Criticism of Plato and the Academy, The John Hopkins Press, Baltimore.

Devereux, D., 1994, "Separation and Immanence in Plato's Theory of Forms", Oxford Studies in Ancient Philosophy, vol. 12, pp. 63-90.

Dooley, W.E., 1989, Alexander of Aphrodisias: On Aristotle, Metaphysics I, Duckworth, Londres.

${ }^{43}$ En la elaboración de este artículo he recibido la ayuda de muchas personas. Quiero agradecer a Ricardo Salles todo el apoyo que me ha brindado antes y durante la realización de este trabajo, del cual ha leído varias versiones y me ha dado numerosos y valiosos comentarios. También agradezco a Axel Barceló, Enrique Hülsz, María Teresa Padilla, José Antonio Robles y a dos dictaminadores de Diánoia por sus muy útiles comentarios a versiones anteriores de este artículo. Finalmente, deseo agradecer el apoyo recibido por parte de los proyectos PAPIIT IN 401301 y CONACyT 40891-H, bajo la responsabilidad de Ricardo Salles, dentro de cuyo marco se desarrolló el presente trabajo. 
Düring, I., 1990, "El escrito sobre las Ideas", en I. Düring, Aristóteles, 2da. ed., trad. B. Navarro del original alemán de 1966, Instituto de Investigaciones Filosóficas-UnAM, México, pp. 385-398.

Fine, G., 1993, On Ideas: Aristotle's Criticism of Plato's Theory of Forms, Clarendon Press, Oxford.

_, 1984, "Separation", Oxford Studies in Ancient Philosophy, vol. 2, pp. 3187.

Frege, G., 1959, The Foundations of Arithmetic, trad. J.L. Austin del original alemán de 1884, Blackwell, Oxford. [Versión en castellano: Conceptografía. Los fundamentos de la aritmética. Otros estudios filosóficos, trad. Hugo Padilla, Instituto de Investigaciones Filosóficas-UnAM, México, 1972.]

Harlfinger, D., 1975 "Edizione critica del testo del 'De Ideis' di Aristotele", en W. Leszl, Il 'De Ideis' di Aristotele, e la teoria platonica delle idee, Olschki, Florencia, pp. 15-39.

Isnardi Parente, M., 1981, "Le Peri Ideôn d'Aristote, Platon ou Xénocrate?", Phronesis, vol. 26, pp. 135-152.

Kahn, C., 1966, "The Greek Verb 'To Be' and the Concept of Being”, Foundations of Language, vol. 2, pp. 245-265.

Kripke, S., 1980, Naming and Necessity, Harvard University Press, Cambridge Massachusetts. [Versión en castellano: El nombrar y la necesidad, 2a. ed., trad. Margarita M. Valdés, Instituto de Investigaciones Filosóficas-UNAM, 2005.]

Lewis, D., 1986, On the Plurality of Worlds, Blackwell, Oxford.

Liddell, H.G., R. Scott y H.S. Jones, 1968, A Greek-English Lexicon, Clarendon Press, Oxford.

Plantinga, A., 1974, The Nature of Necessity, Clarendon Press, Oxford.

Salles, R. y J. Molina, 2004, “iDepende todo lo que hacemos de factores externos? Causalidad externa y causalidad interna en la psicología estoica de las acciones", Noua Tellus, vol. 22, no. 2, pp. 123-177.

Santa Cruz, M.I., M.I. Crespo y S. Di Camillo, 2000, Las críticas de Aristóteles a Platón en el tratado Sobre las Ideas, Eudeba, Buenos Aires.

Vlastos, G., 1987, “'Separation' in Plato”, Oxford Studies in Ancient Philosophy, vol. 5, pp. 187-196.

Recibido el 8 de octubre de 2006; aceptado el 6 de junio de 2007.

Diánoia, vol. LIII, no. 60 (mayo 2008). 